\title{
A Fibrin Matrix Promotes the Differentiation of EMSCs Isolated from Nasal Respiratory Mucosa to Myelinating Phenotypical Schwann-Like Cells
}

\author{
Qian Chen', Zhijian Zhang ${ }^{1, *}$, Jinbo Liư', Qinghua He', Yuepeng Zhou', Genbao Shao', Xianglan Sun', \\ Xudong Cao ${ }^{4}$, Aihua Gong ${ }^{1}$, and Ping Jiang
}

\begin{abstract}
Because Schwann cells perform the triple tasks of myelination, axon guidance and neurotrophin synthesis, they are candidates for cell transplantation that might cure some types of nervous-system degenerative diseases or injuries. However, Schwann cells are difficult to obtain. As another option, ectomesenchymal stem cells (EMSCs) can be easily harvested from the nasal respiratory mucosa. Whether fibrin, an important transplantation vehicle, can improve the differentiation of EMSCs into Schwann-like cells (SLCs) deserves further research. EMSCs were isolated from rat nasal respiratory mucosa and were purified using anti-CD133 magnetic cell sorting. The purified cells strongly expressed HNK-1, nestin, p75 ${ }^{N T R}$, S-100, and vimentin. Using nuclear staining, the MTT assay and Western blotting analysis of the expression of cell-cycle markers, the proliferation rate of EMSCs on a fibrin matrix was found to be significantly higher than that of cells grown on a plastic surface but insignificantly lower than that of cells grown on fibronectin. Additionally, the EMSCs grown on the fibrin matrix expressed myelination-related molecules, including myelin basic protein (MBP), 2',3'cyclic nucleotide 3'-phosphodiesterase (CNPase) and galactocerebrosides (GalCer), more strongly than did those grown on fibronectin or a plastic surface. Furthermore, the EMSCs grown on the fibrin matrix synthesized more neurotrophins compared with those grown on fibronectin or a plastic surface. The expression level of integrin in EMSCs grown on fibrin was similar to that of cells grown on fibronectin but was higher than that of cells grown on a plastic surface. These results demonstrated that fibrin not
\end{abstract}

\footnotetext{
${ }^{1}$ Department of Histology and Embryology, School of Medicine, Jiangsu University, Zhenjiang, China, ${ }^{2}$ Department of Orthopedics, the Third Affiliated Hospital of Suzhou University, Changzhou, China, ${ }^{3}$ School of Pharmacology, Jiangsu University, Zhenjiang, China, ${ }^{4}$ Department of Chemical Engineering, University of Ottawa, Ottawa, Ontario, Canada *Correspondence: zzj@ujs.edu.cn
}

Received 16 June, 2014; revised 8 October, 2014; accepted 19 November, 2014; published online 4 February, 2015

Keywords: fibrin, ectomesenchymal stem cells, myelinating phenotype, Schwann-like cells only promoted EMSC proliferation but also the differentiation of EMSCs into the SLCs. Our findings suggested that fibrin has great promise as a cell transplantation vehicle for the treatment of some types of nervous system diseases or injuries.

\section{INTRODUCTION}

Nervous system injury always results in severe functional impairments due to permanent neurological tissue damage caused by the inability of neural cells to efficiently regenerate. The effectiveness of the available therapeutic strategies is largely unsatisfactory. With the emergence of cell transplantation therapy, the situation is gradually changing because accumulated evidence demonstrates its potential for the treatment of traumatic nervous system injuries (Yazdani et al., 2013). The chosen cell type is one crucial factor in the effectiveness of such a therapy (Zhu et al., 2014).

Schwann cells (SCs) are myelinating and neurotrophic glia found in the peripheral nervous system (PNS), which play important roles in post-injury nerve regeneration through promoting neural regeneration and guiding axon regeneration (Armati and Mathey, 2014). Moreover, transplanting SCs in animal models of sciatic nerve injury and spinal cord injury enhances axonal regeneration and promotes functional recovery (Berrocal et al., 2013; Enomoto et al., 2013; Wang and Xu, 2014). Therefore, SC transplantation to repair nerves has been recommended. However, the application of this approach in clinical practices is restricted by the concomitant donor-site morbidity and the slow growth of these cells in vitro (Tian et al., 2012). To overcome these restrictions, several researchers attempted to obtain differentiated cells resembling SCs from bone marrow stromal cells using various inducing formulae. Unfortunately, the BMSC acquisition procedures are painful for the donor and frequently require general or spinal anesthesia, and the number of harvested BMSCs is low (Wei et al., 2010). Therefore, alternative sources of stem cells must be found.

The lamina propria of the nasal mucosa contains neural crest derived stem cells that can differentiate into cells of ectodermal and mesodermal lineages (Hauser et al., 2012). For this reason, the cells derived from neural crest are called ectomesenchymal stem cells (EMSCs). Because the neural crest is regarded as the forth germ layer and its cells mainly develop into peripheral nervous-system components, its direct descendants, the 
EMSCs, naturally have the propensity to differentiate into SCs (Hall, 2008). Our preliminary study confirmed this speculation. Some of the passaged EMSCs cultured in vitro expressed SC markers, such as p75 ${ }^{\mathrm{NTR}}$, and produced several types of neurotrophins, such as nerve-growth factor (NGF) and brain derived neurotrophic factor (BDNF). These cells were reminiscent of the Schwann-like cells derived from BMSCs and can be considered Schwann-like cells (SLCs). Regrettably, the percentage of SLCs obtained through spontaneous differentiation was low. Several options to enhance the commitment of EMSCs to SLCs must be considered. One convenient option is to apply an effective formula containing neuregulin that is routinely used to induce the differentiation of stem cells to SLCs (Rutten et al., 2012). Co-culturing with Schwann cells is another option for obtaining SLCs from stem cells (Wei et al., 2010). However, neither of these options is satisfactory for application in clinical practice.

The cell scaffold is another important factor in transplantation and it affects the differentiation of stem cells (Gasparotto et al., 2014; Schurmann et al., 2014). Fibrin has received extensive attention in the field of wound healing and has been widely studied for the repair of nervous system injuries (Sharp et al., 2014). A fibrin scaffold containing EMSCs was found to promote histological and behavioral improvements in the rat $\mathrm{SCl}$ model (Liu et al., 2013). It was speculated that fibrin enhanced the differentiation of EMSCs to a myelinating phenotype.

Fibronectin is one of the most commonly used extracellular matrices for cultured stem cells. Fibronectin is known to be particularly important for the growth and differentiation of many cell types (Linsley et al., 2013). However, it is difficult to form three-dimensional scaffolds using fibronectin, and therefore, this molecules is always used to modify other types of matrices (Kang et al., 2014).

In this study, we investigated the effects of fibrin on the spontaneous differentiation of EMSCs into SLCs. The compatibility of EMSCs with fibrin was first studied, and then the phenotypes of EMSCs cultured on a plastic surface, on fibronectin or a fibrin matrix were compared. In addition, the synthesis of neurotrophins and integrin by EMSCs grown on the three substrates was also analyzed.

\section{MATERIALS AND METHODS}

\section{EMSC cultures and purification}

All of the animal procedures were approved by the Jiangsu University Animal Care and Ethics Committee, and the International Guidelines for Animal Research were strictly followed in this study. Primary ectomesenchymal stem cells were isolated from rat respiratory mucosa. Briefly, nasal respiratory mucosa were dissected from the lower one third of the nasal septum of anaesthetized male or female Sprague-Dawley rats weighing $120 \mathrm{~g}-140 \mathrm{~g}$, minced into pieces $\left(0.5-1 \mathrm{~mm}^{3}\right)$ and then incubated in a $0.25 \%$ trypsin solution at $37^{\circ} \mathrm{C}$ for $25 \mathrm{~min}$. After neutralization of the trypsin using fresh DMEM, the cells were dissociated by gentle stirring and were collected by centrifugation at $1000 \times g$ for $5 \mathrm{~min}$. The pellet of dissociated cells was re-suspended in DMEM/F12 (1:1, v/v) medium containing 10\% (v/v) FBS, and then the cells were incubated in plastic flasks in an atmosphere of $5 \% \mathrm{CO}_{2}$ at $37^{\circ} \mathrm{C}$. The medium was changed every 3 days, and the cells were routinely passaged every week.

After the $2^{\text {nd }}$ passage, the cultured cells were purified using immunomagnetic cell sorting. Single cell suspensions of passaged nasal respiratory mucosal cells (approximately $5 \times 10^{7}$ ) were incubated with CD133 immunomagnetic beads (Miltenyi
Biotec, Germany) for $30 \mathrm{~min}$ at $4^{\circ} \mathrm{C}$. A MACS cell-separation column was used to harvest the CD133-positive cells that were attached to the beads. The cell purity was determined by immunofluorescence staining for CD133. Finally, the harvested cells were plated in DMEM/F12 $(1: 1, \mathrm{v} / \mathrm{v})$ medium containing $10 \%(v / v)$ FBS.

The Schwann cell line s42, which is derived from rat sciatic nerves, was purchased from ATCC and served as the positive control for the analysis of myelinating phenotypic markers, integrin, and neurotrophins in EMSCs grown on different substrates.

Fibrin matrix preparation, fibronectin coating and EMSC seeding

In this study, fibrinogen that was purchased from Sigma and fresh rat plasma were used to prepare a fibrin matrix. The fibrinogen was dissolved in DMEM medium at the concentration of $100 \mathrm{mg} / \mathrm{ml}$ and then was mixed with an equal volume of fresh rat plasma to obtain a viscous solution. The solution was apportioned into 24 -well ( $40 \mu \mathrm{l}$ per well), 6 -well (100 $\mu$ l per well) or 96 -well $(20 \mu \mathrm{l}$ per well) plates, which were then incubated at $37^{\circ} \mathrm{C}$ for $30 \mathrm{~min}$; the solution in each well gelled to form a fibrin matrix at a final concentration of fibrinogen of $50 \mathrm{mg} / \mathrm{ml}$.

Rat plasma fibronectin powder (Millipore) was dissolved in 1 $\times$ Tris-buffered saline (TBS) to obtain a $20 \mu \mathrm{g} / \mathrm{ml}$ solution. The fibronectin solution was apportioned into 24 -well ( $1 \mathrm{ml}$ per well), 6 -well $(2 \mathrm{ml}$ per well) or 96 -well $(150 \mu \mathrm{l}$ per well) plates, and the fibronectin was allowed to adsorb to the surfaces at $37^{\circ} \mathrm{C}$ for one hour. The excess fibronectin solution was removed, and the wells were washed twice using TBS prior to cell seeding.

To prepare the EMSCs for plating, the cells were digested using trypsin, centrifuged at $1000 \mathrm{~g}$ for $5 \mathrm{~min}$, and then resuspended at $5 \times 10^{5} \mathrm{cells} / \mathrm{ml}$ in DMEM medium. One-hundredmicroliters of suspended cells (approximately $5 \times 10^{4}$ cells) was transferred to 24 -well or 6-well plates, and then $600 \mu \mathrm{l}$ (24-well plates) or $1.5 \mathrm{ml}$ (6-well plates) of fresh medium was added. Thereafter, the culture medium was changed every 3 days.

\section{Evaluation of cell growth on the fibrin matrix}

Twelve days after seeding the EMSCs on the plastic surfaces, the fibronectin coating surfaces or the fibrin matrix, the cultured cells were fixed and were stained using Hoechst 33342 . The MTT assay was performed to compare the growth of EMSCs on the three substrates. Briefly, EMSCs were seeded at a density of $1 \times 10^{4}$ cells/well in 96-well plates and were incubated $\left(37^{\circ} \mathrm{C}\right.$ and $\left.5 \% \mathrm{CO}_{2}\right)$ for $3,6,9$ or 12 days. An aliquot of an MTT (purchased from Sigma) solution was added to each well and the plate was incubated for $4 \mathrm{~h}$. MTT was metabolized by the mitochondrial dehydrogenases in the living cells into a formazan product, the absorbance of which was measured at $595 \mathrm{~nm}$ using a spectrophotometer ( $\mu$ Quant). To validate the results, immunoblotting to evaluate the expression of biochemical markers, including p21, p16 and PCNA, was also performed 12 days after seeding the EMSCs.

Microscopic evaluation of the fibrin matrix and the EMSCs grown on the matrix

Unloaded fibrin matrices were fixed overnight using 4\% paraformaldehyde and then were dehydrated at room temperature using a graded series of $10-50 \%$ sucrose. Serial sections were prepared using a cryostat and were stained using eosin. Hematoxylin was not used because the sample did not contain cells. The sections were examined and images were captured using a Leica microscope equipped with camera. 
The surface structures of cells grown on the fibrin matrix were examined using a scanning electron microscope (SEM). The fibrin matrix containing EMSCs and the fibrin matrix were fixed using a $2 \%$ phosphate-buffered glutaraldehyde solution and were dehydrated using a graded isopropanol series, followed by gold sputter coating in the manner standard for SEM The prepared samples were observed under an ISI model SX40 SEM (Japan). The unloaded fibrin matrix was processed and observed following the same procedure.

Samples for TEM were fixed using $2.5 \%$ glutaraldehyde (Sigma) for $24 \mathrm{~h}$, embedded, sectioned into ultra-thin sections and mounted on copper grids. The mounted samples were post-stained using $1 \%$ uranyl acetate followed by lead citrate and then were examined using a HITACHI H7650 transmission electron microscope.

\section{Immunocytochemistry and immunoblotting}

Immunofluorescence staining was conducted to determine the characteristics of the purified cells and the cells grown on different substrates. In detail, cultured cells were fixed overnight using $4 \%$ paraformaldehyde at $4^{\circ} \mathrm{C}$. After rinsing twice using PBS, the cells were treated with $0.3 \%$ Triton X-100 in PBS and then blocked using $20 \%$ goat serum. The primary antibodies were diluted using PBS containing $1 \%(\mathrm{w} / \mathrm{v}) \mathrm{BSA}$ and $0.3 \%(\mathrm{v} / \mathrm{v})$ Triton X-100 and applied to the blocked cells overnight at $4^{\circ} \mathrm{C}$. The following primary antibodies were used: as ectomesenchymal cells markers, antibodies directed against CD133 (from Miltenyi Biotec), human natural killer-1 (HNK-1), nestin, p75 ${ }^{\mathrm{NTR}}, \mathrm{S} 100$, and vimentin (1:100, all obtained from Abcam); as markers of myelinating phenotypes, antibodies directed against myelin basic protein (MBP), 2',3'-cyclic nucleotide 3'-phosphodiesterase (CNPase) and galactocerebrosides (GalCer) (1:200, all purchased from Abcam). The samples were rinsed using PBS and then incubated with the appropriate Cy3-conjugated secondary antibodies at $37^{\circ} \mathrm{C}$ for $1 \mathrm{~h}$. At last, all the samples were restained using Hoechst 33342 to label the nuclei. The negative controls were prepared by following the same procedure without using a primary antibody. The fluorescent images were observed and captured using a Leica DM LB2 microscope.

The expression of biomarkers and molecular cell-cycle markers by the purified EMSCs and the phenotypes of the EMSCs grown on different substrates were characterized using Western blotting, as described below. Briefly, the cells were digested using a $0.25 \%$ trypsin solution and were washed several times using PBS. Then, the cells were centrifuged, lysed using a RIPA buffer (AMRESCO) and mixed with SDS-PAGE sample buffer. The proteins were separated by electrophoresis and then were transferred to a PVDF membrane. The blotted membrane was blocked using BSA, incubated with primary antibodies and then with HRP-conjugated secondary antibodies. The labeled bands were detected using an ECL kit (Millipore). Images were captured using an imaging system equipped with a cooled CCD camera (Sage Imaging System, China), according to the manufacturer's instructions. The optical densities of the bands were quantified using ImageJ software $(\mathrm{NIH})$ and then were standardized using the density of the $\beta$-actin bands. The primary antibodies applied for Western blotting include the above-mentioned antibodies directed against CD133, HNK-1, nestin, p75 ${ }^{\mathrm{NTR}}, \mathrm{S} 100$, and vimentin (1:300); a polyclonal rabbit anti-rat p21 antibody, mouse anti-rat p16 and anti-PCNA antibodies (all purchased from Boster, China, 1:200); monoclonal rabbit anti-rat MBP and CNPase antibodies (1:500, Abcam); a polyclonal rabbit anti-rat integrin $\alpha \mathrm{V}$ antibody (1:150, Boster); monoclonal rabbit anti-rat BDNF, NGF, and neurotro phins-3

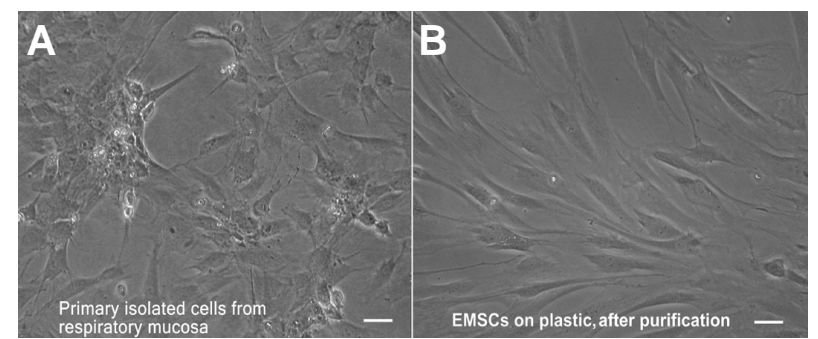

Fig. 1. Morphology of EMSCs grown on a plastic surface. (A) Primary cultured EMSCs grown on a plastic surface; (B) Purified EMSCs grown on a plastic surface. Bar $=5 \mu \mathrm{m}$.

(NT-3) antibodies (1:200, Santa Cruz); and finally, a horseradish peroxidase (HRP) -conjugated monoclonal mouse anti-rat $\beta$-actin antibody (1:10000; Santa Cruz) to stain the loading control protein. The secondary antibodies were HRP-conjugated goat antirabbit or anti-mouse IgGs.

\section{Statistical analysis}

The data were expressed as the mean values \pm the standard error of the mean (SEM).An analysis of variance (ANOVA) was used to analyze the effects of the substrates. Least-significant difference (LSD) multiple-comparison tests were used to compare the mean values of the three groups. A P-value below 0.05 was considered statistically significant.

\section{RESULTS}

Morphology of EMSCs isolated from nasal respiratory mucosa and cultured on a plastic surface

Seven days after isolation, the cultured cells had flat, spindlelike or polygonal shapes with two to four processes (Fig. 1A). With proliferation and the resulting increased level of confluence, the cells contacted one other but did not pile up. After purification, the selected cells largely showed a fibroblast-like morphology resembling the morphology of EMSCs isolated from facial processes (Nie et al., 2014). After the purified cells were passaged several times, they became larger and more flattened (Fig. 1B)

Immunophenotypes of purified EMSCs grown on a plastic surface

To determine the immunophenotypical profiles of the purified cells, immunostaining was performed to assess the expression of various markers, including CD133, HNK-1, nestin, p75 ${ }^{\mathrm{NTR}}, \mathrm{S}$ 100 , and vimentin.

Based on the negative control staining results, as shown in Fig. 2G, all of the fore-mentioned markers were strongly expressed in the cells that were isolated from respiratory mucosa, as shown in Figs. 2A-2F. The results of Western blotting analysis of these markers further confirmed their expression by purified EMSCs (Fig. $2 \mathrm{H}$ ). The purification procedure ensured that a high percentage of CD133-positive cells were present in the cultured population. It is worth mentioning the granular distribution of CD133 on the surface of the EMSCs was consistent with the results of previous studies (Corbeil et al., 2000; Richardson et al., 2004).

Structure of the unloaded fibrin matrix

Under the light microscope, the fibrin matrix showed a mesh- 

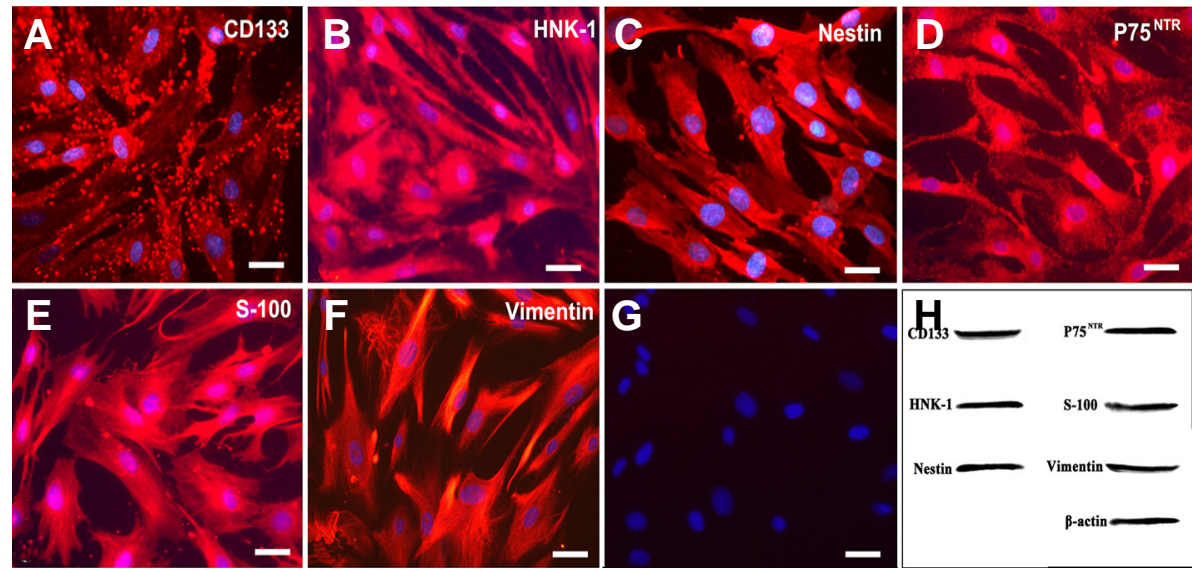

Fig. 2. Expression of CD133, HNK1 , nestin, p75 $75^{\mathrm{NTR}}, \mathrm{S}-100$, and vimentin in purified EMSCs that were grown on a plastic surface and were passaged several times. (G), negative control (primary antibody replaced by PBS); $(\mathrm{H})$, Western blot. Bar $=5 \mu \mathrm{m}$.

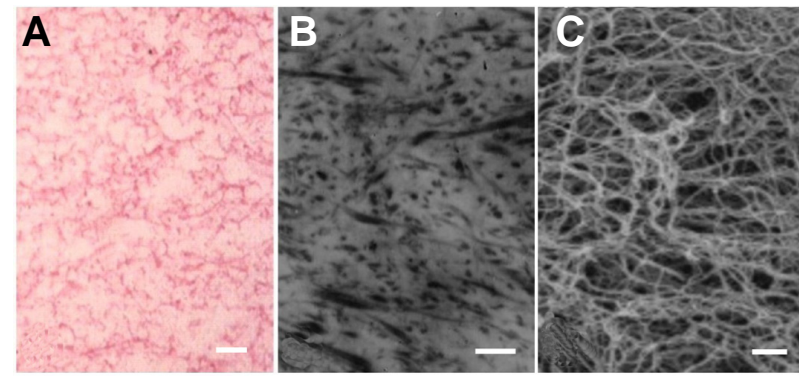

Fig. 3. Morphology of the unloaded fibrin matrix. Structural properties of the fibrin matrix observed using light microscopy (A), TEM $(B)$, and SEM (C). The fibrin matrix possessed a porous structure with an average pore size of approximately $1 \mu \mathrm{m}$. Bar $=1 \mu \mathrm{m}$.

like structure with filaments that were stained red by eosin (Fig. $3 A$ ). Analysis of cross-sectional TEM images showed that the diameter of the filaments in the matrix was approximately 0.05 $\mu \mathrm{m}$ and that the distance between adjacent filaments was much greater than the thickness of a filament (Fig. 3B). The SEM images demonstrated the sponge-like $3 \mathrm{D}$ structures of the matrix, with connected filaments forming numerous pores with diameters of between $0.5-1 \mu \mathrm{m}$ (Fig. 3C). The porous network of the matrix and the high affinity of fibrin filaments for various growth factors made this matrix suitable for the promotion of cellular adhesion and the conservation of water and nutrient.

Growth and proliferation of EMSCs grown on a plastic surface, fibronectin or the fibrin matrix

Although the EMSCs could be cultured for several months on a plastic surface by passaging them, it was still unclear whether these cells could survive on the fibrin matrix. Therefore, the EMSCs were cultured on the fibrin matrix for 12 days. As shown in Fig. 4C, Hoechst 33342-stained nuclei of the EMSCs were distributed over the entire fibrin matrix. Some cells wrapped around the fibrin filaments and formed a cylindrical structure (Fig. 4C). The density of nuclei observed on the fibrin matrix was significantly higher than that on a plastic surface but was insignificantly lower than that on fibronectin (Fig. 4D).

To evaluate the proliferation of EMSCs grown on the fibrin matrix, the MTT assay was utilized. As shown in Fig. 4E, we discerned that the rate of growth of cells cultured on the fibrin matrix was more rapid than that on a plastic surface but was slower than that on fibronectin.

Western blotting to analyze the expression of cell-cycle markers, including p21, p16 and PCNA, indicated that the proliferation rate of EMSCs on fibrin was greater than that on of cells grown on a plastic surface (Figs. 4F and 4G).

\section{Morphology of EMSCs grown on the fibrin matrix}

Twelve days after seeding on fibrin matrix, many of the EMSCs had attained the typical bipolar or tripolar morphology reminiscent of Schwann cells (Fig. 5C). If the culture period was prolonged, some of the cells could even be observed to cling closely to the filaments that had partially detached from the matrix and to dangle with the filaments within the culture medium.

The SEM images showed that compared with the cells grown on a plastic surface, the EMSCs grown on fibronectin were more flattened (Fig. 5E versus 5D) and that those grown on the fibrin matrix were slimmer (Fig. 5F versus 5D). Moreover, these EMSCs formed a better meshwork structure than did the cells grown on a plastic surface (Fig. 5F versus 5D).

\section{Myelinating phenotypes of EMSCs grown on the fibrin matrix}

Twelve days after being seeded on the fibrin matrix, the shape of the EMSCs had changed from flattened to a bipolar or multipolar morphology, reminiscent of Schwann cells. These SLCs expressed myelination-related molecules, such as MBP, CNPase and GalCer, more strongly than did the EMSCs grown on a plastic surface or on fibronectin (Fig. 6). The results of Western blotting confirmed the higher levels of MBP and CNPase in the EMSCs grown on the fibrin matrix compared with those grown on a plastic surface or fibronectin (Fig. 7). The levels of MBP and CNPase expression by the EMSCs grown on the fibrin matrix were similar to those of the $\mathrm{s} 42$ cells, as shown in Fig. 7.

\begin{abstract}
Expression of neurotrophins and integrin by EMSCs grown on the different substrates

Because Schwann cells can synthesize neurotrophins, we evaluated the expression of neurotrophins in the EMSCs and compared the levels of neurotrophins expression by EMSCs grown on the fibrin matrix, fibronectin and a plastic surface. As shown in Fig. 7, the levels of BDNF, NGF and NT-3 expression of EMSCs grown on the fibrin matrix were significantly higher than those of the EMSCs grown on a plastic surface or on fibronectin and were
\end{abstract}



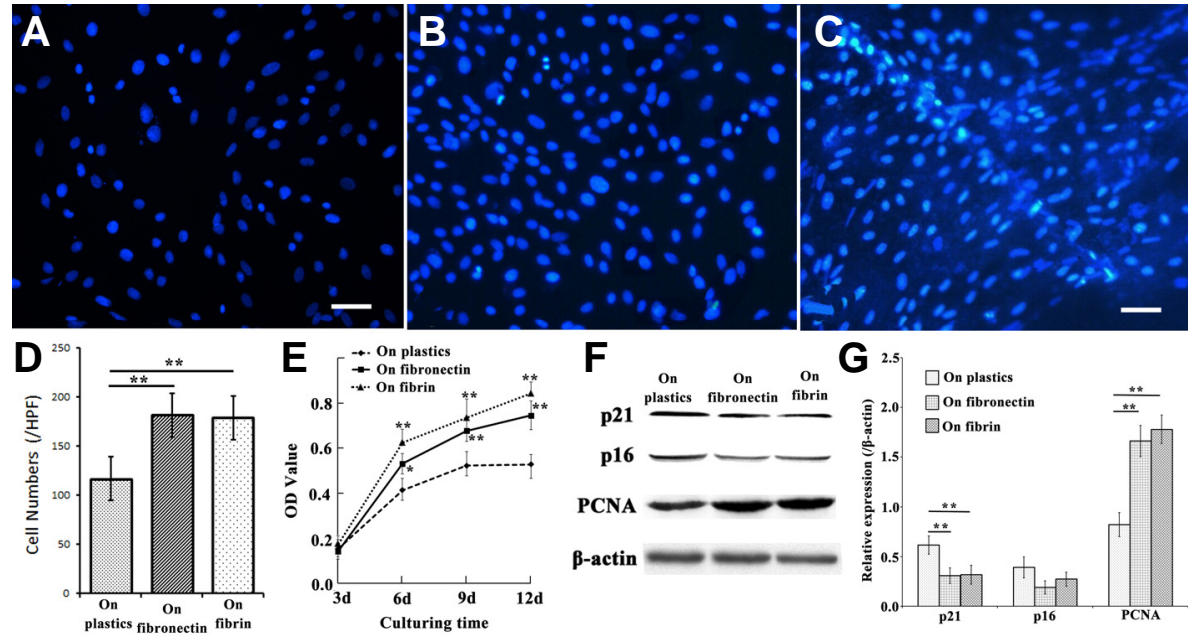

$\boldsymbol{G}_{25}$

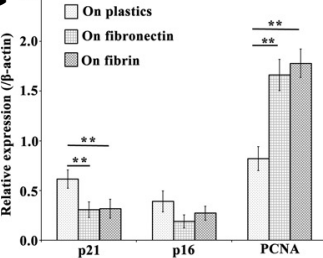

Fig. 4. Proliferation of EMSCs grown on a plastic surface, fibronectin or the fibrin matrix. Nuclei of EMSCs grown on a plastic surface (A), fibronectin (B), or the fibrin matrix (C). (D) Cell density of EMSCs cultured for 12 days; $(E)$ The results of the MTT assay showed the different rates of proliferation of EMSCs grown on the three substrates. $(\mathrm{F})$ Representative Western blot of cellcycle markers p21, p16, and PCNA, with $\beta$-actin serving as the loading control; (G) quantitative analysis of the Western blot; each bar represents the mean value \pm SEM of measurements made in four independent replicates. Bar $=10 \mu \mathrm{m} ;{ }^{*} \mathrm{P}$ $<0.05,{ }^{* \star} \mathrm{P}<0.01$.
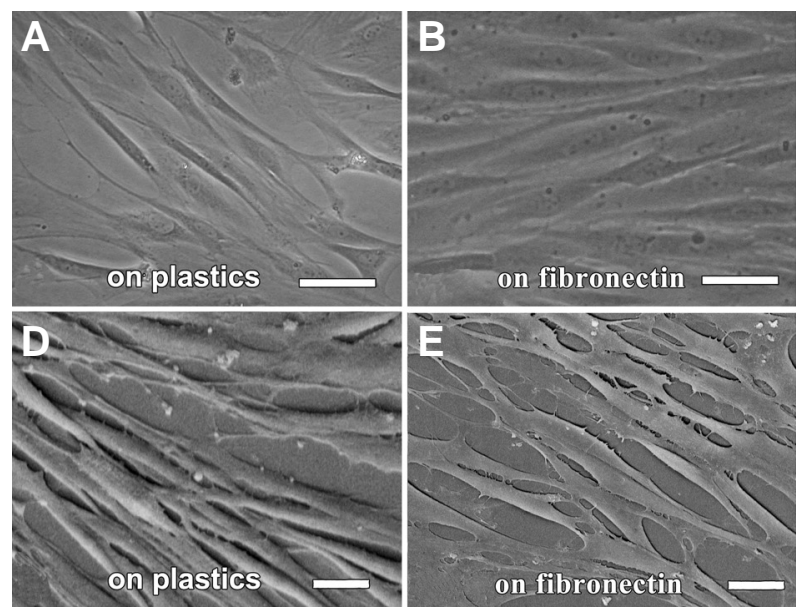
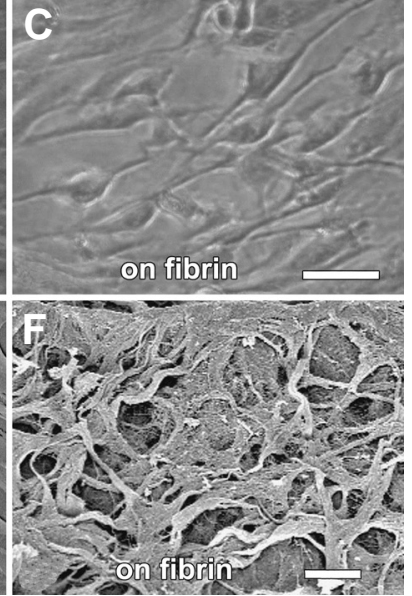

Fig. 5. Different morphologies of EMSCs grown on a plastic surface, fibronectin or the fibrin matrix. The EMSCs grown on the fibrin matrix had thin processes reminiscent of those of Schwann cells. SEM showed that the EMSCs and the fibrin matrix formed a better meshwork. (A-C) phase contrast light microscopy; (D-F) SEM. Bar $=10$ $\mu \mathrm{m}$. even insignificantly higher than those of the $\$ 42$ Schwann cell line. The expression level of integrin $\alpha \mathrm{V}$ of the EMSCs grown on fibrin was higher than that of the EMSCs grown on a plastic surface or of the s42 cells but was insignificantly lower than that of the EMSCs grown on fibronectin.

\section{DISCUSSION}

Extensive studies of olfactory ensheathing cells (OECs), one type of adult stem cell obtained from the olfactory mucosa, have produced abundant results, whereas the stem cells of the nasal respiratory mucosa were less investigated until recently. In this study, the stem cells of the murine nasal respiratory mucosa, which were defined as ectomesenchymal stem cells (EMSCs), were isolated and the effects of a fibrin matrix on their spontaneous glial differentiation were investigated.

The strong level of HNK-1, S-100, p75 $5^{\mathrm{NTR}}$ and vimentin in the cultured cells was consistent with the properties of the EMSCs obtained from the first branchial arch of mouse embryos (Yan et al., 2006). Although the presence of $p 75^{\text {NTR }}$ could not distinguish EMSCs from OECs, the general and homogenous expression of HNK-1 confirmed that the cultured cells were
EMSCs because HNK-1 exists on EMSCs other than OECs (Lin et al., 2006). Because both p $75^{\mathrm{NTR}}$ and HNK-1 are markers of neural crest cells, the EMSCs investigated in this study may have originated from the cranial neural crest (Betters et al., 2010). The fact that cranial neural crest cells can differentiate into various types of cells and form different tissues may explain the tendency of EMSCs to differentiate into SLCs in vitro (Jacob et al., 2014).

The fibrin matrix that polymerized from the mixture of rat plasma and commercially available rat fibrinogen exhibited a porous structure in which the size of the pores could be regulated by adjusting the final concentration of fibrinogen (Linnes et al., 2007). A final fibrinogen concentration of $50 \mathrm{mg} / \mathrm{ml}$ was chosen based on the results of a preliminary study of the proliferation of cells grown on matrices constructed from fibrinogen solutions of various concentrations. At this specific concentration, the proliferation rate of EMSCs grown on the fibrin matrix was insignificantly lower than that of EMSCs grown on fibronectin but significantly higher than that of EMSCs grown on a plastic surface.

The results of the proliferation assay appear somewhat paradoxical because the stiffness of a fibrin matrix is much lower than that of a plastic surface, and the cell proliferation rate in a 

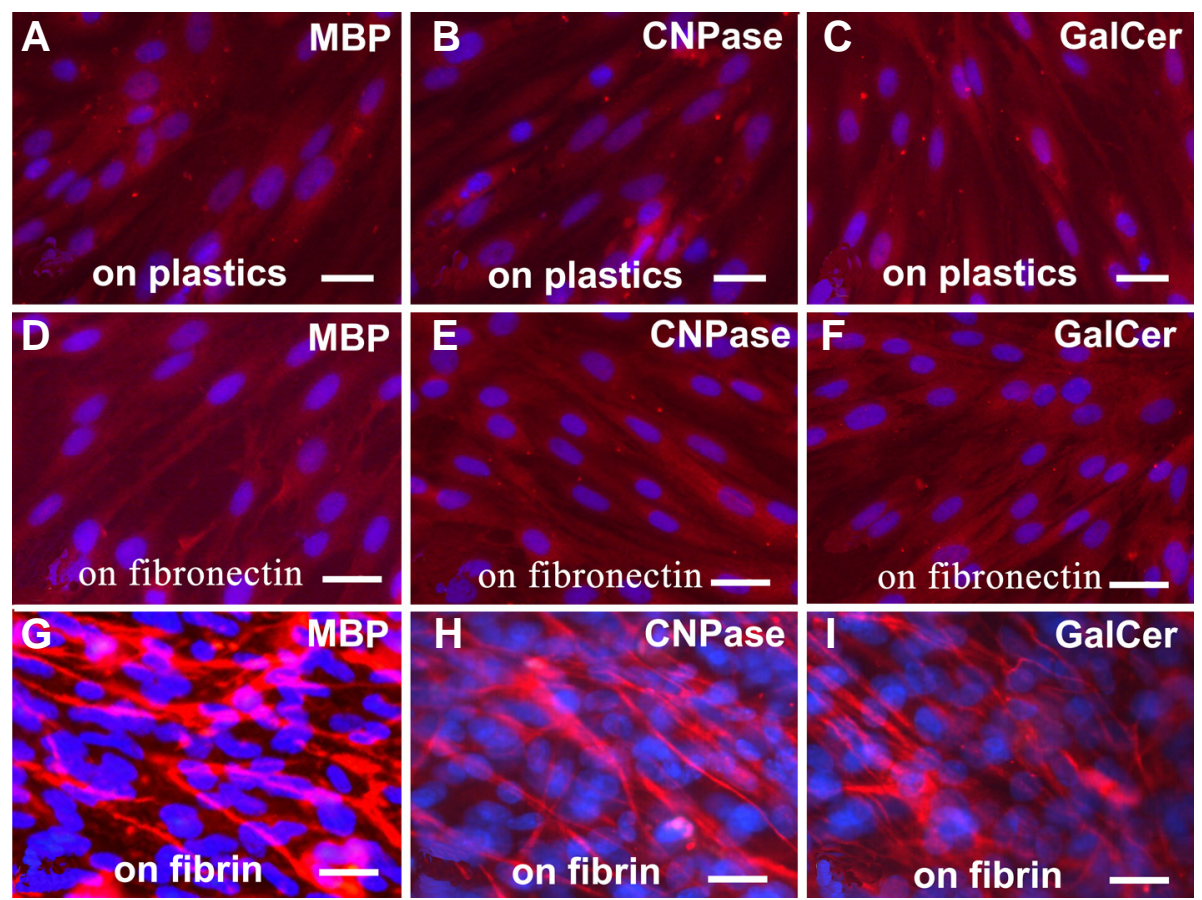

Fig. 6. Differential expression of myelination-related molecules by EMSCs grown on a plastic surface, fibronectin or the fibrin matrix. The EMSCs grown on the fibrin matrix showed stronger expression of MBP, CNPase and GalCer than did the cells grown on a plastic surface or on fibronectin. Bar $=5$ $\mu \mathrm{m}$.
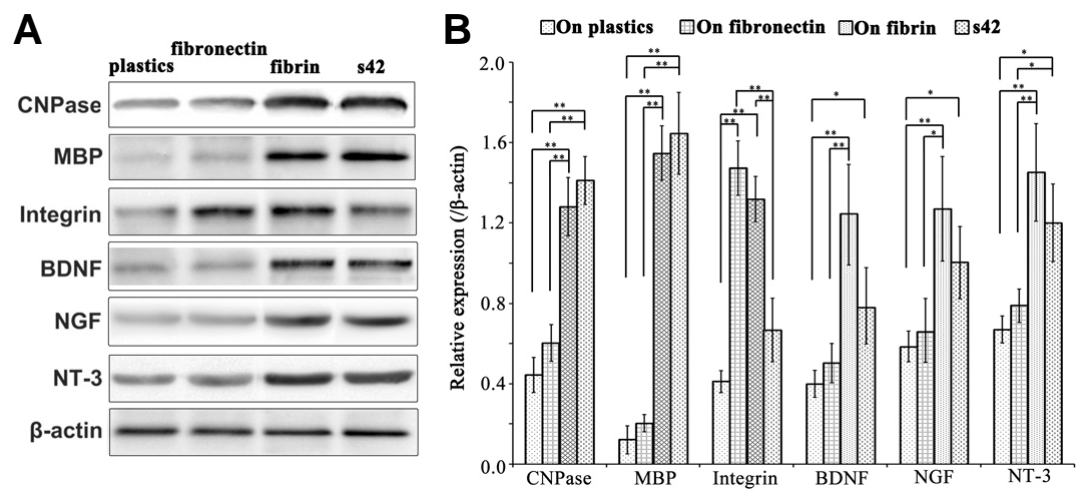

Fig. 7. Levels of expression of myelinationrelated molecules and neurotrophins of EMSCs grown on the three substrates and the rat Schwann cell line s42. (A) Representative Western blot in which $\beta$-actin served as the loading control. (B) Quantitative analysis of the Western blot; each bar represents the mean value \pm SEM of measurements made on five replicates. ${ }^{\star} \mathrm{P}<0.05,{ }^{* \star} \mathrm{P}<0.01$.

compliant matrix environment is reported to be lower than that on a stiff matrix (Hadjipanayi et al., 2009). We speculated that fibrin, despite its unfavorable stiffness, promoted proliferation not only by providing stronger biological stimuli, such as arginine-glycine-aspartic acid (RGD) sequences, than does a plastic surface but also by providing a larger surface area (Riopel et al., 2013; Weis et al., 2013). Because both fibrin and fibronectin contain RGD sequences, the expansion of the surface area of the former may not justify the negative effect of its relatively low stiffness on cell proliferation based on the comparison between cell growth on fibrin and fibronectin. Similar to the results regarding cell proliferation, the higher level of integrin expression in the EMSCs grown on fibrin compared with that of the cells grown on a plastic surface may be induced by the RGD sequences and greater surface area, whereas the lower level of integrin expression in the EMSCs grown on fibrin compared with that of the cells grown on fibronectin may have been caused by the lower stiffness of fibrin.
In addition, the shapes of the cells grown on the fibrin matrix were generally slimmer than those of the cells grown on a plastic surface or on fibronectin. Taken together with the finding that integrin-dependent focal adhesions increased cellular tension, which may have caused the cells to become flattened, the higher level of integrin expression in the EMSCs grown on fibrin compared with that of the cells grown on a plastic surface appears not to be the cause of the slimmer shapes of the EMSCs grown on fibrin (Natale et al., 2014). The slim shape of the EMSCs grown on fibrin may have been determined by the long thin fibrils present in the fibrin matrix, as shown in Fig. $3 \mathrm{C}$, rather than integrin signaling. These slim differentiated cells are reminiscent of the Schwann cells surrounding the axons of neurons. To some extent, these cells can be regarded as SLCs.

Because remyelination is an important issue in the repair of CNS injuries, whether the SLCs derived from EMSCs would facilitate myelination is important. The enzyme CNPase is the earliest myelination-specific protein expressed in both myelinating 
oligodendrocytes and Schwann cells. It is regarded as one indicator of the potential of cells to produce myelin, rather than an indication of actual myelination (Radtke et al., 2011). Similar to CNPase, MBP is another major component of CNS myelin and is found in Schwann cells (Lutz et al., 2014). The third marker of the myelinating phenotype is the oligodendrocyte- and Schwann cellspecific glycosphingolipid GalCer, which exists in the lipid bilayer of the myelin membrane of the CNS and PNS (Kondo et al., 2005). Immunofluorescence staining demonstrated a stronger level of CNPase, MBP and GalCer expression in the EMSCs grown on the fibrin matrix compared with those grown on a plastic surface or on fibronectin, which was confirmed by Western blotting. These results are consistent with the finding that fibrin matrices facilitated the production of myelin-associated glycoproteins in the lesioned spinal cord (Lu et al., 2012).

It was reported that Schwann cell-based myelination requires the interaction of the RGD peptides that help laminin to form a basement membrane and integrin molecules on Schwann cells (McKee et al., 2012; Ness et al., 2013). Because both fibrin and lamin contain RGD peptides, it is natural to hypothesize that the promotion of myelination by fibrin might be due to the interaction between RGD and integrin, which is present on the EMSCs (Fowlkes et al., 2013). However, the results rule out the possibility that the differentiation of EMSCs into SLCs induced by fibrin was caused merely by the RGD sequences and integrin signaling because the EMSCs grown on fibronectin did not differentiate like those grown on the fibrin matrix.

In contrast, accumulating evidence suggests that the elasticity or the stiffness of matrices can determine the differentiation route taken by various types of stem cells (Lu et al., 2014). The mechanical interaction between the attachment sites of cells and the elastic response of the substrate was reported to be a pivotal determinant of cellular morphology (De Santis et al., 2011). Another possible factor affecting EMSC differentiation into SLCs is the topography of the fibrin matrix because the matrix topography was reported to direct stem-cell differentiation (Christopherson et al., 2009; Oh et al., 2009; Lu et al., 2014). The thin fibrils containing RGD sequences in the fibrin matrix might guide the differentiation of EMSCs into SLCs (Chen et al., 2014). Furthermore, the elasticity and topography of the fibrin matrix are both totally different from those of fibronectin. Therefore, these two factors might contribute to the differentiation of EMSCs into SLCs on the fibrin matrix. Further studies are required to clarify the myelination capacities of these cells through co-culturing them with neurons in vitro or applying them to treat nervous system injuries in vivo.

The significance of Schwann cells in cell therapies to treat nervous system injuries is ardently appreciated not only due to their myelinating ability but also due to their synthesis of neurotrophins, which enhance the survival and regeneration of neurons.

Neurotrophins are a family of proteins that are responsible for the growth and survival of neurons. Numerous studies have addressed the application of neurotrophins, including nerve growth factor (NGF), brain derived neurotrophic factor (BDNF), and neurotrophin-3 (NT-3), for the repair of nervous system injuries or treatment of neurodegenerative diseases, such as Parkinson's disease (Sullivan and Toulouse, 2011; Zhang et al., 2012). It is worthwhile to examine the effect of fibrin on the production of neurotrophins. As shown using Western blotting, the cells grown on the fibrin matrix synthesized more neurotrophins compared with those grown on a plastic surface or on fibronectin. Together with the morphological and phenotypical changes observed in the cells grown on the fibrin matrix, the higher levels of neurotrophins suggested that multiple facets of the EMSCs were altered by the microenvironment if we regard the fibrin matrix as a component of an extracellular matrix and hence a component of the cellular environment. Understanding the fascinating interactions between stem cells and a fibrin matrix-based microenvironment will require further studies.

In summary, a fibrin matrix promoted the differentiation of EMSCs to myelinating cells, and also enhanced their level of neurotrophin synthesis. These findings indicated the possible dual beneficial roles of fibrin as a transplantation vehicle and a myelination promoter in cellular therapies for the treatment of degenerative nervous diseases or injuries.

\section{ACKNOWLEDGMENTS}

This study was supported by the National Natural Science Foundation of China (grants No. 30570981, No. 30571878 and No. 81170573). This study was also supported by the fund of Jiangsu University for talents.

\section{REFERENCES}

Armati, P.J., and Mathey, E.K. (2014). Clinical implications of Schwann cell biology. J. Peripher. Nerv. Syst. 19, 14-23.

Berrocal, Y.A., Almeida, V.W., Gupta, R., and Levi, A.D. (2013) Transplantation of Schwann cells in a collagen tube for the repair of large, segmental peripheral nerve defects in rats. J. Neurosurg. 119, 720-732.

Betters, E., Liu, Y., Kjaeldgaard, A., Sundstrom, E., and GarciaCastro, M.I. (2010). Analysis of early human neural crest development. Dev. Biol. 344, 578-592.

Chen, P., Cescon, M., and Bonaldo, P. (2014). The role of collagens in peripheral nerve myelination and function. Mol. Neurobiol. 2014, 1-10.

Christopherson, G.T., Song, H., and Mao, H.Q. (2009). The influence of fiber diameter of electrospun substrates on neural stem cell differentiation and proliferation. Biomaterials 30, 556-564.

Corbeil, D., Roper, K., Hellwig, A., Tavian, M., Miraglia, S., Watt, S.M., Simmons, P.J., Peault, B., Buck, D.W., and Huttner, W.B. (2000). The human AC133 hematopoietic stem cell antigen is also expressed in epithelial cells and targeted to plasma membrane protrusions. J. Biol. Chem. 275, 5512-5520.

De Santis, G., Lennon, A.B., Boschetti, F., Verhegghe, B., Verdonck, P., and Prendergast, P.J. (2011). How can cells sense the elasticity of a substrate? An analysis using a cell tensegrity model. Eur. Cell. Mater. 22, 202-213.

Enomoto, M., Bunge, M.B., and Tsoulfas, P. (2013). A multifunctional neurotrophin with reduced affinity to p75 enhances transplanted Schwann cell survival and axon growth after spinal cord injury. Exp. Neurol. 248C, 170-182.

Fowlkes, V., Wilson, C.G., Carver, W., and Goldsmith, E.C. (2013) Mechanical loading promotes mast cell degranulation via RGDintegrin dependent pathways. J. Biomech. 46, 788-795.

Gasparotto, V.P., Landim-Alvarenga, F.C., Oliveira, A.L., Simoes, G.F., Lima-Neto, J.F., Barraviera, B., and Ferreira, R.S., Jr. (2014). A new fibrin sealant as a three-dimensional scaffold candidate for mesenchymal stem cells. Stem Cell Res. Ther. 5, 78.

Hadjipanayi, E., Mudera, V., and Brown, R.A. (2009). Close dependence of fibroblast proliferation on collagen scaffold matrix stiffness. J. Tissue Eng. Regen. Med. 3, 77-84.

Hall, B.K. (2008). The neural crest and neural crest cells: discovery and significance for theories of embryonic organization. J. Biosci. 33, 781-793.

Hauser, S., Widera, D., Qunneis, F., Muller, J., Zander, C., Greiner, J., Strauss, C., Luningschror, P., Heimann, P., Schwarze, H., et al. (2012). Isolation of novel multipotent neural crest-derived stem cells from adult human inferior turbinate. Stem Cells Dev. $21,742-756$

Jacob, C., Lotscher, P., Engler, S., Baggiolini, A., Varum Tavares, S., Brugger, V., John, N., Buchmann-Moller, S., Snider, P.L., Conway, S.J., et al. (2014). HDAC1 and HDAC2 control the specification of neural crest cells into peripheral glia. J. Neurosci. 
34, 6112-6122.

Kang, B.J., Kim, H., Lee, S.K., Kim, J., Shen, Y., Jung, S., Kang K.S., Im, S.G., Lee, S.Y., Choi, M., et al. (2014). Umbilical-cordblood-derived mesenchymal stem cells seeded onto fibronectinimmobilized polycaprolactone nanofiber improve cardiac function. Acta Biomater. 10, 3007-3017.

Kondo, Y., Wenger, D.A., Gallo, V., and Duncan, I.D. (2005) Galactocerebrosidase-deficient oligodendrocytes maintain stable central myelin by exogenous replacement of the missing enzyme in mice. Proc. Natl. Acad. Sci. USA 102, 18670-18675.

Lin, Y., Yan, Z., Liu, L., Qiao, J., Jing, W., Wu, L., Chen, X., Li, Z., Tang, W., Zheng, X., et al. (2006). Proliferation and pluripotency potential of ectomesenchymal cells derived from first branchial arch. Cell Prolif. 39, 79-92.

Linnes, M.P., Ratner, B.D., and Giachelli, C.M. (2007). A fibrinogenbased precision microporous scaffold for tissue engineering. $\mathrm{Bi}$ omaterials $28,5298-5306$.

Linsley, C., Wu, B., and Tawil, B. (2013). The effect of fibrinogen, collagen type $\mathrm{I}$, and fibronectin on mesenchymal stem cell growth and differentiation into osteoblasts. Tissue Eng. Part A 19, 1416-1423.

Liu, J., Chen, Q., Zhang, Z., Zheng, Y., Sun, X., Cao, X., Gong, A., Cui, Y., He, Q., and Jiang, P. (2013). Fibrin scaffolds containing ectomesenchymal stem cells enhance behavioral and histological improvement in a rat model of spinal cord injury. Cells Tissues Organs 198, 35-46.

Lu, P., Wang, Y., Graham, L., McHale, K., Gao, M., Wu, D., Brock J., Blesch, A., Rosenzweig, E.S., Havton, L.A., et al. (2012). Long-distance growth and connectivity of neural stem cells after severe spinal cord injury. Cell 150, 1264-1273.

Lu, D., Luo, C., Zhang, C., Li, Z., and Long, M. (2014). Differential regulation of morphology and stemness of mouse embryonic stem cells by substrate stiffness and topography. Biomaterials 35, 3945-3955.

Lutz, D., Loers, G., Kleene, R., Oezen, I., Kataria, H., Katagihallimath, N., Braren, I., Harauz, G., and Schachner, M. (2014). Myelin basic protein cleaves cell adhesion molecule L1 and promotes neuritogenesis and cell survival. J. Biol. Chem. 289, 13503-13518.

McKee, K.K., Yang, D.H., Patel, R., Chen, Z.L., Strickland, S., Takagi, J., Sekiguchi, K., and Yurchenco, P.D. (2012). Schwann cel myelination requires integration of laminin activities. J. Cell Sci. $125,4609-4619$.

Natale, C.F., Ventre, M., and Netti, P.A. (2014). Tuning the materialcytoskeleton crosstalk via nanoconfinement of focal adhesions. Biomaterials 35, 2743-2751.

Ness, J.K., Snyder, K.M., and Tapinos, N. (2013). Lck tyrosine kinase mediates beta1-integrin signalling to regulate Schwann cell migration and myelination. Nat. Commun. 4, 1912.

Nie, X., Xing, Y., Deng, M., Gang, L., Liu, R., Zhang, Y., and Wen, $X$. (2014). Ecto-mesenchymal stem cells from facial process: potential for muscle regeneration. Cell Biochem. Biophys. 70, 615-622.

Oh, S., Brammer, K.S., Li, Y.S., Teng, D., Engler, A.J., Chien, S. and Jin, S. (2009). Stem cell fate dictated solely by altered nanotube dimension. Proc. Natl. Acad. Sci. USA 106, 2130-2135.
Radtke, C., Sasaki, M., Lankford, K.L., Gallo, V., and Kocsis, J.D. (2011). CNPase expression in olfactory ensheathing cells. J. Biomed. Biotechnol. 2011, 608496.

Richardson, G.D., Robson, C.N., Lang, S.H., Neal, D.E., Maitland, N.J., and Collins, A.T. (2004). CD133, a novel marker for human prostatic epithelial stem cells. J. Cell Sci. 117, 3539-3545.

Riopel, M., Stuart, W., and Wang, R. (2013). Fibrin improves beta (INS-1) cell function, proliferation and survival through integrin $\alpha v \beta 3$. Acta Biomater. 9, 8140-8148.

Rutten, M.J., Janes, M.A., Chang, I.R., Gregory, C.R., and Gregory, K.W. (2012). Development of a functional schwann cell phenotype from autologous porcine bone marrow mononuclear cells for nerve repair. Stem Cells Int. 2012, 738484.

Schurmann, M., Wolff, A., Widera, D., Hauser, S., Heimann, P., Hutten, A., Kaltschmidt, C., and Kaltschmidt, B. (2014). Interaction of adult human neural crest-derived stem cells with a nanoporous titanium surface is sufficient to induce their osteogenic differentiation. Stem Cell Res. 13, 98-110.

Sharp, K.G., Yee, K.M., and Steward, O. (2014). A re-assessment of long distance growth and connectivity of neural stem cells after severe spinal cord injury. Exp. Neurol. 257, 186-204.

Sullivan, A.M., and Toulouse, A. (2011). Neurotrophic factors for the treatment of Parkinson's disease. Cytokine Growth Factor Rev. 22, 157-165.

Tian, X., Wang, S., Zhang, Z., and Lv, D. (2012). Rat bone marrowderived Schwann-like cells differentiated by the optimal inducers combination on microfluidic chip and their functional performance. PLoS One 7, e42804.

Wang, X., and Xu, X.M. (2014). Long-term survival, axonal growthpromotion, and myelination of Schwann cells grafted into contused spinal cord in adult rats. Exp. Neurol. 261, 308-319.

Wei, Y., Gong, K., Zheng, Z., Liu, L., Wang, A., Zhang, L., Ao, Q., Gong, Y., and Zhang, X. (2010). Schwann-like cell differentiation of rat adipose-derived stem cells by indirect co-culture with Schwann cells in vitro. Cell Prolif. 43, 606-616.

Weis, S., Lee, T.T., Del Campo, A., and Garcia, A.J. (2013). Dynamic cell-adhesive microenvironments and their effect on myogenic differentiation. Acta. Biomater. 9, 8059-8066.

Yan, Z., Lin, Y., Jiao, X., Li, Z., Wu, L., Jing, W., Qiao, J., Liu, L., Tang W., Zheng, X., et al. (2006). Characterization of ectomesenchymal cells isolated from the first branchial arch during multilineage differentiation. Cells Tissues Organs 183, 123-132.

Yazdani, S.O., Hafizi, M., Zali, A.R., Atashi, A., Ashrafi, F., Seddighi, A.S., and Soleimani, M. (2013). Safety and possible outcome assessment of autologous Schwann cell and bone marrow mesenchymal stromal cell co-transplantation for treatment of patients with chronic spinal cord injury. Cytotherapy 15, 782-791.

Zhang, J., Shi, Q., Yang, P., Xu, X., Chen, X., Qi, C., Zhang, J., Lu, H., Zhao, B., Zheng, P., et al. (2012). Neuroprotection of neurotrophin-3 against focal cerebral ischemia/reperfusion injury is regulated by hypoxia-responsive element in rats. Neuroscience 222, 1-9.

Zhu, T., Tang, Q., Gao, H., Shen, Y., Chen, L., and Zhu, J. (2014). Current status of cell-mediated regenerative therapies for human spinal cord injury. Neurosci. Bull. 30, 671-682. 